 \\ MOUNTaiNS \\ Mathematical Publications
}

\section{EXISTENCE OF ASYMPTOTICALLY PERIODIC SOLUTIONS OF SCALAR VOLTERRA DIFFERENCE EQUATIONS}

\author{
Josef Diblík — Miroslava RŮŽičKová - Ewa Schmeidel
}

\begin{abstract}
There is used a version of Schauder's fixed point theorem to prove the existence of asymptotically periodic solutions of a scalar Volterra difference equation. Along with the existence of asymptotically periodic solutions, sufficient conditions for the nonexistence of such solutions are derived. Results are illustrated on examples.
\end{abstract}

\section{Introduction}

We consider a Volterra difference equation

$$
x(n+1)=a(n)+b(n) x(n)+\sum_{i=0}^{n} K(n, i) x(i),
$$

where

$$
n \in \mathbb{N}:=\{0,1,2, \ldots\}, a, b, x: \mathbb{N} \rightarrow \mathbb{R}, K: \mathbb{N} \times \mathbb{N} \rightarrow \mathbb{R}
$$

and $\mathbb{R}$ denotes the set of all real numbers. By a solution of equation (1) we mean a sequence $x: \mathbb{N} \rightarrow \mathbb{R}$ whose terms satisfy (1) for every $n \in \mathbb{N}$. Throughout this paper we will assume that sequences $a$ and $K$ are not identically equal to zero.

We will also adopt the customary notations

$$
\sum_{i=k+s}^{k} \mathcal{O}(i)=0, \quad \prod_{i=k+s}^{k} \mathcal{O}(i)=1,
$$

2000 Mathematics Subject Classification: 39A11, 39A10.

Keywords: asymptotically periodic solution, asymptotic behavior of solutions, Volterra difference equation.

The first and the second authors have been supported by the project APVV-0700-07 of Slovak Research and Development Agency and by Grant No. 1/0090/09 of the Grant Agency of Slovak Republic (VEGA). 


\section{JOSEF DIBLÍK - MIROSLAVA RŮŽIČKOVÁ - EWA SCHMEIDEL}

where $k$ is an integer, $s$ is a positive integer and " $\mathcal{O}$ " denotes the function considered independently of whether it is defined for the arguments indicated or not.

Definition 1. Let $\omega$ be a positive integer. The sequence $y: \mathbb{N} \rightarrow \mathbb{R}$ is called $\omega$ -periodic if $y(n+\omega)=y(n)$ for all $n \in \mathbb{N}$. The sequence $y$ is called asymptotically $\omega$-periodic if there exist two sequences $u, v: \mathbb{N} \rightarrow \mathbb{R}$ such that $u$ is $\omega$-periodic, $\lim _{n \rightarrow \infty} v(n)=0$ and $y(n)=u(n)+v(n)$ for all $n \in \mathbb{N}$.

The background for discrete Volterra equations can be found in the well known monograph [1] by Agarwal, as well as in Elaydi [3] and Kocić and L a d as [7.

Uniform asymptotic stability in linear Volterra difference equations was studied by $\mathrm{Elaydi}$ and $\mathrm{Murakami}$ in [4. Periodic and asymptotically periodic solutions of linear difference equations were investigated, e.g., by A g a r w al and $\mathrm{P}$ opend a in [2, and by Popenda and Schmeidel in [9, 10].

In [5] and 6], Furumochi considered the behavior of solutions of the following classes of Volterra difference equations

$$
x(n+1)=a(n)-\sum_{i=0}^{n} D(n, i, x(i))
$$

and

$$
x(n+1)=p(n)-\sum_{i=-\infty}^{n} P(n, i, x(i)),
$$

and their inter-relations. Boundedness, attractivity, and convergence of solutions were investigated.

\section{Asymptotically periodic solutions}

In this section, sufficient conditions for the existence of an asymptotically $\omega$-periodic solution of equation (11) are given. The following version of Schauder's fixed point theorem, which can be found in [8], will be used to prove the main result of this paper.

Lemma 1. Let $\Omega$ be a Banach space and $S$ its nonempty, closed and convex subset, and let $T$ be a continuous mapping such that $T(S)$ is contained in $S$, and the closure $\overline{T(S)}$ is compact. Then $T$ has a fixed point in $S$. 
Let $\omega$ be a positive integer and $b: \mathbb{N} \rightarrow \mathbb{R} \backslash\{0\}$ be $\omega$-periodic. Then we define an $\omega$-periodic function $\beta: \mathbb{N} \rightarrow \mathbb{R}$ as

$$
\beta(n)=\left\{\begin{array}{lll}
\prod_{j=0}^{n-1} \frac{1}{b(j)} & \text { if } & n \geq 1 \\
\beta(\omega) & \text { if } & n=0
\end{array}\right.
$$

Further we define

$$
m:=\min \{|\beta(1)|,|\beta(2)|, \ldots,|\beta(\omega)|\}
$$

and

$$
M:=\max \{|\beta(1)|,|\beta(2)|, \ldots,|\beta(\omega)|\} .
$$

TheOREM 1 (Main result). Let $\omega$ be a positive integer and $b: \mathbb{N} \rightarrow \mathbb{R} \backslash\{0\}$ be $\omega$-periodic. Assume that

$$
\begin{gathered}
\prod_{i=0}^{\omega-1} b(i)=1 \\
\sum_{i=0}^{\infty}|a(i)|<\infty
\end{gathered}
$$

and

$$
\sum_{j=0}^{\infty} \sum_{i=0}^{j}|K(j, i)|<\frac{m}{M}
$$

Then, for any nonzero constant $c$, there exists an asymptotically $\omega$-periodic solution $x$ of (11) such that

$$
x(n)=u(n)+v(n), \quad n \in \mathbb{N}
$$

with

$$
u(n):=c \prod_{k=0}^{n^{*}} b(k) \quad \text { and } \quad \lim _{n \rightarrow \infty} v(n)=0
$$

where $n^{*}$ is the remainder of dividing $n-1$ by $\omega$.

Pr o of. We note that $n^{*}=n-1-\omega\left[\frac{n-1}{\omega}\right]$ where the function $[\cdot]$ is the greatest integer function.

From the $\omega$-periodicity of sequence $b$, the definition of $\beta$ by (2), and the property (3) we have

$$
\beta(n) \in\{\beta(1), \beta(2), \ldots, \beta(\omega)\}
$$

for any $n \in \mathbb{N}$. Thus,

$$
m \leq|\beta(n)| \leq M
$$


for any $n \in \mathbb{N}$. Let $c>0$. We set

$$
\alpha(0):=\frac{M \sum_{i=0}^{\infty}|a(i)|+\frac{c M}{m} \sum_{j=0}^{\infty} \sum_{i=0}^{j}|K(j, i)|}{1-\frac{M}{m} \sum_{j=0}^{\infty} \sum_{i=0}^{j}|K(j, i)|}
$$

and

$$
\alpha(n):=M \sum_{i=n}^{\infty}|a(i)|+\frac{(c+\alpha(0)) M}{m} \sum_{j=n}^{\infty} \sum_{i=0}^{j}|K(j, i)|,
$$

for $n \geq 1$. It is easy to see that

$$
\lim _{n \rightarrow \infty} \alpha(n)=0
$$

We show, moreover, that

$$
\alpha(n) \leq \alpha(0)
$$

for any $n \in \mathbb{N}$. Let us first remark that

$$
\alpha(0)=M \sum_{i=0}^{\infty}|a(i)|+\frac{(c+\alpha(0)) M}{m} \sum_{j=0}^{\infty} \sum_{i=0}^{j}|K(j, i)| .
$$

Then, due to the convergence of series (4), (5), the inequality

$$
\begin{aligned}
\alpha(0) & =M \sum_{i=0}^{\infty}|a(i)|+\frac{(c+\alpha(0)) M}{m} \sum_{j=0}^{\infty} \sum_{i=0}^{j}|K(j, i)| \\
& \geq M \sum_{i=n}^{\infty}|a(i)|+\frac{(c+\alpha(0)) M}{m} \sum_{j=n}^{\infty} \sum_{i=0}^{j}|K(j, i)|=\alpha(n)
\end{aligned}
$$

obviously holds for every $n \in \mathbb{N}$ and (10) is proved.

Let $B$ be the Banach space of all real bounded sequences $z: \mathbb{N} \rightarrow \mathbb{R}$ equipped with the usual supremum norm. We define a subset $S \subset B$ as

$$
S:=\{z(n) \in B: c-\alpha(0) \leq z(n) \leq c+\alpha(0), \quad n \in \mathbb{N}\} .
$$

It is not difficult to prove that $S$ is a nonempty, bounded, convex, and closed subset of $B$.

Let us define a mapping $T: S \rightarrow B$ as follows

$$
(T z)(n)=c-\sum_{i=n}^{\infty} a(i) \beta(i+1)-\sum_{j=n}^{\infty} \sum_{i=0}^{j} \frac{\beta(j+1)}{\beta(i)} K(j, i) z(i)
$$


for any $n \in \mathbb{N}$. We will prove that the mapping $T$ has a fixed point in $B$. We first show that $T(S) \subset S$. Indeed, if $z \in S$, then

$$
|z(n)-c| \leq \alpha(0) \quad \text { for } \quad n \in \mathbb{N},
$$

and, by (8) and (11), we have

$$
\begin{aligned}
|(T z)(n)-c| & \leq M \sum_{i=n}^{\infty}|a(i)|+\frac{(c+\alpha(0)) M}{m} \sum_{j=n}^{\infty} \sum_{i=0}^{j}|K(j, i)| \\
& =\alpha(n) \leq \alpha(0) .
\end{aligned}
$$

Next we prove that $T$ is continuous. Let $z^{(p)}$ be a sequence in $S$ such that $z^{(p)} \rightarrow z$ as $p \rightarrow \infty$. Because $S$ is closed, $z \in S$. Now, by (15), (8) and (111), we get

$$
\begin{aligned}
\left|\left(T z^{(p)}\right)(n)-(T z)(n)\right| & =\left|\sum_{j=n}^{\infty} \sum_{i=0}^{j} \frac{\beta(j+1)}{\beta(i)} K(j, i)\left(z^{(p)}(n)-z(n)\right)\right| \\
& \leq \frac{M}{m} \cdot \frac{m}{M} \cdot \sup _{i \geq 0}\left|z^{(p)}(i)-z(i)\right|, \quad n \in \mathbb{N} .
\end{aligned}
$$

Therefore

$$
\left|\left(T z^{(p)}\right)(n)-(T z)(n)\right| \leq \sup _{i \geq 0}\left|z^{(p)}(i)-z(i)\right|, \quad n \in \mathbb{N}
$$

and

$$
\lim _{p \rightarrow \infty}\left|T z^{(p)}-T z\right|=0
$$

This means that $T$ is continuous.

To prove that $\overline{T(S)}$ is compact, we take $\varepsilon>0$. Then, from (9), we conclude that there exists $n_{\varepsilon} \in \mathbb{N}$ such that $\alpha(n)<\varepsilon$, for $n \geq n_{\varepsilon}$. We cover the segment $[c-\alpha(0), c+\alpha(0)]$ with a finite number $k_{\varepsilon}$ of intervals each having a length of $\varepsilon$. Let the points $c_{k_{\varepsilon}}$ be the centres of the $\varepsilon$-length intervals. We conclude that, for an arbitrarily small $\varepsilon>0$, we can collect a finite set of intervals with centres at $c_{k_{\varepsilon}}$ and with radii $\varepsilon / 2$ which covers $\overline{T(S)}$. Hence $\overline{T(S)}$ is compact.

By Schauder's fixed point theorem (see Lemma 1), there exists a $z \in S$ such that

Thus

$$
z(n)=(T z)(n) \quad \text { for } n \in \mathbb{N} .
$$

$$
\begin{aligned}
z(n)=c & -\sum_{i=n}^{\infty} a(i) \beta(i+1) \\
& -\sum_{j=n}^{\infty} \sum_{i=0}^{j} \frac{\beta(j+1)}{\beta(i)} K(j, i) z(i) \quad \text { for any } n \in \mathbb{N} .
\end{aligned}
$$




\section{JOSEF DIBLÍK - MIROSLAVA RŮŽIČKOVÁ - EWA SCHMEIDEL}

Due to (9) and (12), for fixed point $z \in S$ of $T$, we have

$$
\lim _{n \rightarrow \infty}|z(n)-c|=\lim _{n \rightarrow \infty}|(T z)(n)-c| \leq \lim _{n \rightarrow \infty} \alpha(n)=0
$$

or, equivalently,

$$
\lim _{n \rightarrow \infty} z(n)=c .
$$

Finally, we will show that there exists a connection of the fixed point $z \in S$ with the existence of asymptotically $\omega$-periodic solution of (1). Considering (13) for $z(n+1)$ and $z(n)$, we get

$$
\Delta z(n)=a(n) \beta(n+1)+\sum_{i=0}^{n} \frac{\beta(n+1)}{\beta(i)} K(n, i) z(i), \quad n \in \mathbb{N} .
$$

Hence, by (2) (taking into account that $\beta(0)=\beta(\omega)=1$ in view of (3) ), we have

$$
\begin{aligned}
z(n+1)-z(n)= & a(n) \beta(n+1)+\frac{\beta(n+1)}{\beta(0)} K(n, 0) z(0) \\
& +\sum_{i=1}^{n} \frac{\beta(n+1)}{\beta(i)} K(n, i) z(i) \\
= & a(n) \prod_{k=0}^{n} \frac{1}{b(k)}+\left(\prod_{k=0}^{n} \frac{1}{b(k)}\right) K(n, 0) z(0) \\
& +\sum_{i=1}^{n}\left(\prod_{k=i}^{n} \frac{1}{b(k)}\right) K(n, i) z(i), \quad n \in \mathbb{N} .
\end{aligned}
$$

Putting

$$
z(n)=\left(\prod_{k=0}^{n-1} \frac{1}{b(k)}\right) x(n), \quad n \in \mathbb{N}
$$

in (15), we get equation (1) since

$$
\begin{aligned}
\frac{x(n+1)}{\prod_{k=0}^{n} \frac{1}{b(k)}}-\frac{x(n)}{\prod_{k=0}^{n-1} \frac{1}{b(k)}}= & a(n) \prod_{k=0}^{n} \frac{1}{b(k)}+\left(\prod_{k=0}^{n} \frac{1}{b(k)}\right) K(n, 0)\left(\prod_{k=0}^{-1} \frac{1}{b(k)}\right) x(0) \\
& +\sum_{i=1}^{n}\left(\prod_{k=i}^{n} \frac{1}{b(k)}\right) K(n, i)\left(\prod_{k=0}^{i-1} \frac{1}{b(k)}\right) x(i), \quad n \in \mathbb{N}
\end{aligned}
$$

yields

$$
x(n+1)=a(n)+b(n) x(n)+K(n, 0) x(0)+\sum_{i=1}^{n} K(n, i) x(i), \quad n \in \mathbb{N} .
$$


Consequently, $x$ defined by (16) is a solution of (11). From (14) and (16), we obtain

$$
\left(\prod_{k=0}^{n-1} \frac{1}{b(k)}\right) x(n)=z(n)=c+o(1)
$$

for $n \rightarrow \infty$ (where $o(1)$ is the Landau order symbol). Hence

$$
x(n)=c \prod_{k=0}^{n-1} b(k)+\left(\prod_{k=0}^{n-1} b(k)\right) o(1), \quad n \rightarrow \infty .
$$

From (3) we get

$$
\prod_{k=0}^{n-1} b(k)=\prod_{k=0}^{n^{*}} b(k)
$$

The proof is complete since the sequence $\left\{\prod_{k=0}^{n^{*}} b(k)\right\}$ is $\omega$-periodic and due to properties of Landau order symbols we have

$$
\left(\prod_{k=0}^{n^{*}} b(k)\right) o(1)=o(1), \quad n \rightarrow \infty .
$$

If $c<0$, the proof, which we omit, can be carried out in a manner similar to the one used above if $x$ is changed to $-x$.

Remark 1. Tracing the proof of Theorem 1 we see that it remains valid even in the case of $c=0$. Then there exists an asymptotically " $\omega$ "-periodic solution $x$ of (11) as well. The formula (6) reduces to

$$
x(n)=v(n)=o(1), \quad n \in \mathbb{N} .
$$

From the point of view of Definition 1, we can consider this case as follows. We set (as a singular case) $u \equiv 0$ with an arbitrary (possibly other than " $\omega$ ") period and with $v=o(1)$ for $n \rightarrow \infty$.

In the following example, a sequence $b$ is 1 -periodic. Then it is 2-periodic, too. By virtue of Theorem 1 there exists a 2-periodic solution of the equation in question.

EXAmple 1. Put

$$
\begin{aligned}
& a(n)=(-1)^{n+1} \cdot \frac{1}{3 \cdot 2^{n+3}}+\frac{53}{48 \cdot 2^{2 n}}+\frac{1}{2^{3 n+4}}, \\
& b(n) \equiv-1 \quad \text { and } \quad K(n, i)=\frac{2^{i}}{4^{n+2}}
\end{aligned}
$$

in (1). We consider the sequence $b$ as a 2-periodic sequence and put $\omega=2$. Obviously, $m=M=1$ and

$$
\sum_{j=0}^{\infty} \sum_{i=0}^{j} K(j, i)=\sum_{j=0}^{\infty} \sum_{i=0}^{j} \frac{2^{i}}{4^{j+2}}=\sum_{j=0}^{\infty} \frac{2^{j+1}-1}{4^{j+2}}=\frac{1}{6}<1 .
$$




\section{JOSEF DIBLÍK - MIROSLAVA RŮŽIČKOVÁ - EWA SCHMEIDEL}

Then all the assumptions of Theorem 1 are satisfied and (by (6) (17) ) there exists an asymptotically 2-periodic solution

$$
x(n)=u(n)+v(n), \quad n \in \mathbb{N}
$$

of the equation (11) where

$$
u(n)=c \prod_{k=0}^{n^{*}} b(k)=c \prod_{k=0}^{n^{*}}(-1)=c(-1)^{n}, \quad \lim _{n \rightarrow \infty} v(n)=0 .
$$

Indeed, a sequence

$$
x(n)=c(-1)^{n}+\frac{1}{4^{n}}
$$

with $c=1$ is such a solution.

\section{Nonexistence of asymptotically periodic solutions}

Finally, we present sufficient conditions for the nonexistence of asymptotically periodic solution of (1) satisfying some auxiliary conditions.

Let $x(n)=u(n)+v(n)$ be an asymptotically periodic solution of (11) such that the sequence $u$ is $\omega$-periodic and $\lim _{n \rightarrow \infty} v(n)=0$.

TheOREM 2. If sequences $a: \mathbb{N} \rightarrow \mathbb{R}$ and $b: \mathbb{N} \rightarrow \mathbb{R}$ are bounded and there exists a positive integer $\omega$ such that

$$
K(n, i)=K(n+\omega, i+\omega)
$$

for all $n, i \in \mathbb{N}$, then the equation (1) does not have any asymptotically $\omega$-periodic solution $x(n)=u(n)+v(n)$ such that

$$
\sum_{i=0}^{\omega-1} K(\omega-1, i) u(i) \neq 0
$$

and

$$
\sum_{i=0}^{\infty}|v(i)|<\infty
$$

P r o of. Suppose, on the contrary, that assumptions of Theorem 2 are satisfied and there exists an asymptotically $\omega$-periodic solution $x$ of equation (1) which satisfies conditions (18) and (19). Without loss of generality we may assume that

$$
\sum_{i=0}^{\omega-1} K(\omega-1, i) u(i)>0 \text {. }
$$


From (17) we have

$$
K(n, i)=K\left(n-\omega\left[\frac{n}{\omega}\right], i-\omega\left[\frac{i}{\omega}\right]\right)=K\left((n+1)^{*},(i+1)^{*}\right) .
$$

Because $x(n)=u(n)+v(n)$, from (17) and the $\omega$-periodicity of the sequence $u$, we have

$$
\begin{aligned}
\sum_{i=0}^{n} K(n, i) x(i)= & \sum_{i=0}^{n} K\left((n+1)^{*},(i+1)^{*}\right) u\left((i+1)^{*}\right) \\
& +\sum_{i=0}^{n} K\left((n+1)^{*},(i+1)^{*}\right) v(i) \\
= & {\left[\frac{n}{\omega}\right] \cdot \sum_{i=0}^{\omega-1} K(\omega-1, i) u(i) } \\
& +\sum_{i=0}^{(n+1)^{*}} K\left((n+1)^{*}, i\right) u(i) \\
& +\sum_{i=0}^{n} K\left((n+1)^{*},(i+1)^{*}\right) v(i) .
\end{aligned}
$$

By (20)

$$
\limsup _{n \rightarrow \infty}\left[\frac{n}{\omega}\right] \cdot \sum_{i=0}^{\omega-1} K(\omega-1, i) u(i)=\infty .
$$

We remark that the sum

$$
\sum_{i=0}^{(n+1)^{*}} K\left((n+1)^{*}, i\right) u(i)
$$

is bounded (for $n \rightarrow \infty$ ) and there exists a positive constant $K^{* *}$ such that

$$
\left|K\left((n+1)^{*},(i+1)^{*}\right)\right| \leq K^{* *}
$$

for all $n, i \in \mathbb{N}$. Then

$$
\left|K\left((n+1)^{*},(i+1)^{*}\right)\right||v(i)| \leq K^{* *}|v(i)|
$$

and, by (19), the series

$$
\sum_{i=0}^{\infty} K\left((n+1)^{*},(i+1)^{*}\right) v(i)
$$

is absolutely convergent. Thus

$$
\limsup _{n \rightarrow \infty} \sum_{i=0}^{n} K(n, i) x(i)=\infty
$$




\section{JOSEF DIBLÍK - MIROSLAVA RŮŽIČKOVÁ - EWA SCHMEIDEL}

Rewriting (11), we get

$$
x(n+1)-a(n)-b(n) x(n)=\sum_{i=0}^{n} K(n, i) x(i),
$$

where the left-hand side of the above equation is bounded while the right-hand side is unbounded. This contradiction completes the proof.

Remark 2. We will emphasize the necessity of (18) in Theorem 2, If

$$
\sum_{i=0}^{\omega-1} K(\omega-1, i) u(i)=0
$$

then (1) can have an asymptotically $\omega$-periodic solution.

Let, e.g., $K(j, i)=\left(1+(-1)^{i}\right) / 2$. Then, taking sequences $a$ and $b$ in (1) in a proper manner, the equation (1) will have an asymptotically 4 -periodic solution $x(n)=u(n)+v(n)$ with 4-periodic function $u(n):=(0,1,0,2, \ldots)$. In this case

$$
\sum_{i=0}^{\omega-1} K(\omega-1, i) u(i)=\sum_{i=0}^{\omega-1} \frac{1+(-1)^{i}}{2} u(i)=1 \cdot 0+0 \cdot 1+1 \cdot 0+0 \cdot 2=0
$$

and (18) does not hold. Then

$$
\limsup _{n \rightarrow \infty}\left[\frac{n}{\omega}\right] \cdot \sum_{i=0}^{\omega-1} K(\omega-1, i) u(i)=0
$$

and we do not get the final contradiction in the proof of Theorem 2

\section{REFERENCES}

[1] AGARWAL, R. P.: Difference Equations and Inequalities. Theory, Methods, and Applications (2nd ed.), in: Pure Appl. Math., Vol. 228, Marcel Dekker, Inc., New York, 2000.

[2] AGARWAL, R. P.-POPENDA, J.: Periodic solutions of first order linear difference equations, Math. Comput. Modelling 22 (1995), 11-19.

[3] ELAYDI, S. N.: An Introduction to Difference Equations (3rd ed.), Undergrad. Texts Math., Springer-Verlag, New York, 2005.

[4] ELAYDI, S. N.-MURAKAMI, S.: Uniform asymptotic stability in linear Volterra difference equations, J. Difference Equ. Appl. 3 (1998), 203-218.

[5] FURUMOCHI, T.: Periodic solutions of Volterra difference equations and attractivity, Nonlinear Anal. 47 (2001), 4013-4024.

[6] FURUMOCHI, T.: Asymptotically periodic solutions of Volterra difference equations, Vietnam J. Math. 30 (2002), 537-550.

[7] KOCIĆ, V. L.-LADAS, G.: Global Behavior of Nonlinear Difference Equations of Higher Order with Applications, in: Math. Appl., Vol. 256., Kluwer Acad. Publ., Dordrecht, 1993.

[8] MUSIELAK, J.: Wstep do Analizy Funkcjonalnej, PWN, Warszawa, 1976. (In Polish) 


\section{EXISTENCE OF ASYMPTOTICALLY PERIODIC SOLUTIONS}

[9] POPENDA, J.-SCHMEIDEL, E.: On the asymptotically periodic solution of some linear difference equations, Arch. Math. (Brno) 35 (1999), 13-19.

[10] POPENDA, J.-SCHMEIDEL, E.: Asymptotically periodic solution of some linear difference equations, Facta Univ. Ser. Math. Inform. 14 (1999), 31-40.

Received December 8, 2008

Josef Diblik

Miroslava Růžičková

Department of Mathematics

Faculty of Science

University of Žilina

Univerzitná 8215/1

SK-010-26 Žilina

SLOVAKIA

E-mail: josef.diblik@fpv.uniza.sk miroslava.ruzickova@fpv.uniza.sk

Ewa Schmeidel

Institut of Mathematics

Faculty of Electrical Engineering

Poznań University of Technology

Piotrowo 3 a

PL-60-965 Poznań

POLAND

E-mail: ewa.schmeidel@put.poznan.pl 